D urante un viaje de recolección por el estado de Veracruz en marzo de 2005, tuvimos la oportunidad de obtener material de Tectaria xmichleriana (D.C.Eaton) Lellinger (Tectariaceae) en el cerro Zapote del Pleito, rancho El Milagro, colonia Nueva Tabasqueña, Municipio de Las Choapas, Veracruz (17 $51^{\prime} 07^{\prime}$ ' N, 9401'59” O, 30 m s.n.m). Éste constituye el primer registro del taxón para México. La localidad es un manchón de selva alta perennifolia de aproximadamente un kilómetro cuadrado, situado en un conjunto de lomas y rodeado por potreros. Se encontró el helecho híbrido (Mendoza R. 900) creciendo junto con sus especies parentales, Tectaria incisa Cav. (Mendoza R. 899) y Tectaria panamensis (Hook.) R.M.Tryon et A.F.Tryon (Mendoza $R$. 908). El material de respaldo se encuentra depositado en el Herbario Metropolitano "Ramón Riba y Nava Esparza" (UAMIZ), de la Universidad Autónoma Metropolitana, Iztapalapa.

Tectaria $\times$ michleriana $[=\times$ Pleuroderris michleriana (D.C. Eaton) Maxon], ha sido descrita varias veces con diez nombres distintos incluyendo el basiónimo arriba citado. Los sinónimos son Aspidium tatei (Baker) Diels, Hypoderris seemanni Prentice, H. heteroneuroides H.Christ, H. marginalis Fourn., H. adnata Fourn., Lindsaea michleriana D.C.Eaton, Phegopteris tatei (Baker) Salomon, Polypodium tatei Baker y Tectaria tatei (Baker) C.Chr. Es un helecho híbrido que se conocía en Mesoamérica únicamente de Guatemala, Nicaragua, Costa Rica y Panamá (Wagner et al., 1972, 1978; Moran, 1995; Lellinger, 2003; Mickel y Smith, 2004). De acuerdo con Mickel y Smith (2004); esta planta podría encontrarse en cualquier sitio en el que coexistan

\title{
Primer ReGISTRO EN MÉXICO DE TECTARIA XMICHLERIANA, TECTARIACEAE
}

\author{
Aniceto Mendoza-Ruiz, Blanca Pérez-García \\ Y RICARDO VALDEZ
}

Universidad Autónoma Metropolitana-Iztapalapa, Departamento de Biología, Área de Botánica Estructural y Sistemática Vegetal, Av. San Rafael Atlixco 186, Col. Vicentina, Iztapalapa 09340, México, D.F., México. Correo-e: amr@xanum.uam.mx

sus dos progenitores Tectaria incisa (Morton, 1966) y Tectaria panamen sis $[=$ Dictyoxiphium panamense Hook.] (Tryon y Tryon, 1981). En el caso de México este helecho se encuentra en el estado de Veracruz, aunque posiblemente también se distribuya en los estados de Oaxaca y Chiapas.

T. Xmichleriana es un helecho terrestre que mide de 1 a $1.5 \mathrm{~m}$ de altura. Presenta un rizoma rastrero y escamoso; sus hojas poseen una lámina pinnatífida a 1-pinnada basalmente; con la parte media apical pinnatífida y los márgenes marcadamente irregulares; las pinnas tienen venación areolada; las areolas con venillas incluidas. Los soros son redondos a alargados, con un indusio insertado lateralmente, y están dispuestos de manera irregular en la parte media de la pinna, submarginales a marginales (cuadro 1, figuras 1-2); sus esporas son monoletes, de color pardo claro, con un perisporio reticulado; el número cromosómico $2 \mathrm{n}=80$ (Wagner et al., 1978).

Este taxón es un híbrido entre
Tectaria incisa y T. panamensis. Este último es un helecho terrestre, de hojas dimorfas con lámina entera, nervaduras areoladas, nérvulos libres incluidos, soros submarginales, lineares, indusio entero y número cromosómico $2 \mathrm{n}=80$ (cuadro 1, figuras 3-4), en tanto que $T$. incisa es también una planta terrestre, de hojas 1pinnadas, 3-10 pares de pinnas; soros dispuestos en dos series entre las nervaduras laterales principales, indusio circular unido lateralmente y número cromosómico $2 \mathrm{n}=80$ (cuadro 1, figuras 5-6). Moran (1995) y Mickel y Smith (2004) mencionan que esta última especie es extremadamente variable en lo que concierne a la división de la lámina y al número de pares de pinnas.

\section{Agradecimientos}

Los autores agradecen a Adolfo Espejo y Ana Rosa López-Ferrari por las valiosas sugerencias hechas al manuscrito, así como a los árbitros Mónica Palacios y uno anónimo, y al editor Jorge Meave, por sus comen- 
tarios hechos para enriquecer este trabajo.

\section{Literatura citada}

Lellinger D.B. 2003. Nomenclatural and taxonomic notes on the pteridophytes of Costa Rica, Panama, and Colombia, III. American Fern Journal 93:146-151.

Mickel J.T. y Smith A.R. 2004. The pteridophytes of Mexico. Memoirs of the New York Botanical Garden 88:11054.

Moran R.C. 1995. Tectariaceae. En:
Moran R.C. y Riba R. Eds. Flora Mesoamericana, Psilotaceae a Salviniaceae, pp. 195-210, Instituto de Biología, Universidad Nacional Autónoma de México, México, D.F.

Morton C.V. 1996. The Mexican species of Tectaria. American Fern Journal 56:120-137.

Tryon R. y Tryon A. 1981. Taxonomic and nomenclatural notes on ferns. Rhodora 83:133-137.

Wagner W.H., Wagner F.S. y GómezPignataro L.D. 1972. The Central American fern genus Pleuroderris. American Journal of Botany 59:677.
Wagner W.H., Wagner F.S. y GómezPignataro L.D. 1978. The singular origin of a Central American fern, Pleuroderris michleriana. Biotropica 10:254-264.

Fecha de recepción: 21 de octubre de 2005

Versión corregida: 3 de mayo de 2006

Aceptado: 3 de mayo de 2006

Cuadro 1. Comparación de caracteres de Tectaria ×michleriana con sus progenitores.

\begin{tabular}{|c|c|c|c|}
\hline Caracteres & T. $\times$ michleriana & T. incisa & T. panamensis \\
\hline Distribución geográfica & México (Veracruz) a Colombia & Cosmopolita & México a Colombia \\
\hline Forma de las hojas & Monomorfas, algo extendidas & Monomorfas, extendidas & Dimorfas, erectas \\
\hline Tamaño de la hoja & De 1 a $1.5 \mathrm{~m}$ & De 0.7 a $1.7 \mathrm{~m}$ & De 0.4 a $1.10 \mathrm{~m}$ \\
\hline Rizoma & Rastrero & Erecto & Semierecto a erecto \\
\hline Lámina & $\begin{array}{l}\text { Simple, lobada pinnatífida a } \\
\text { pinnada irregularmente }\end{array}$ & 1-pinnadas & Simple \\
\hline Margen de la lámina & Entero a ondulado & Ondulado & Entero \\
\hline Textura de la lámina & Gruesa & Herbácea & Coriácea \\
\hline Forma de la lámina & $\begin{array}{l}\text { Linear, lanceolada a } \\
\text { estrechamente triangular }\end{array}$ & $\begin{array}{l}\text { Triangular a } \\
\text { oblongo-triangular }\end{array}$ & Linear \\
\hline Base de la lámina & $\begin{array}{l}\text { Bases de los lobos o pinnas } \\
\text { cortamente pecioladas, con } \\
\text { el ala decurrente, rara o } \\
\text { completamente contraídas } \\
\text { en la base }\end{array}$ & $\begin{array}{l}\text { Largamente peciolado, } \\
\text { base de las pinnas } \\
\text { peciolada }\end{array}$ & $\begin{array}{l}\text { Casi sésiles, la lámina } \\
\text { gradualmente reducida }\end{array}$ \\
\hline Venación & $\begin{array}{l}\text { Areolada con venillas libres } \\
\text { incluidas }\end{array}$ & $\begin{array}{l}\text { Areolada con venillas } \\
\text { libres incluidas }\end{array}$ & $\begin{array}{l}\text { Areolada con venillas } \\
\text { libres incluidas }\end{array}$ \\
\hline Soros & $\begin{array}{l}\text { Redondos, elongados o } \\
\text { alargados con indusio redondo } \\
\text { a oblongo }\end{array}$ & $\begin{array}{l}\text { Redondos con indusio } \\
\text { circular }\end{array}$ & $\begin{array}{l}\text { Lineares y continuos, } \\
\text { con indusio entero }\end{array}$ \\
\hline Posición de los soros & $\begin{array}{l}\text { Marginal o abaxial, raramente } \\
\text { entre la costa y el margen }\end{array}$ & $\begin{array}{l}\text { Abaxial en dos hileras } \\
\text { entre la costa y el margen } \\
\text { de la pinna }\end{array}$ & $\begin{array}{l}\text { Marginales, a lo largo } \\
\text { de la lámina }\end{array}$ \\
\hline Esporas & Monoletes y abortivas & Monoletes, normales & Monoletes, normales \\
\hline Número cromosómico & $2 n=80$ & $2 n=80$ & $2 n=80$ \\
\hline
\end{tabular}



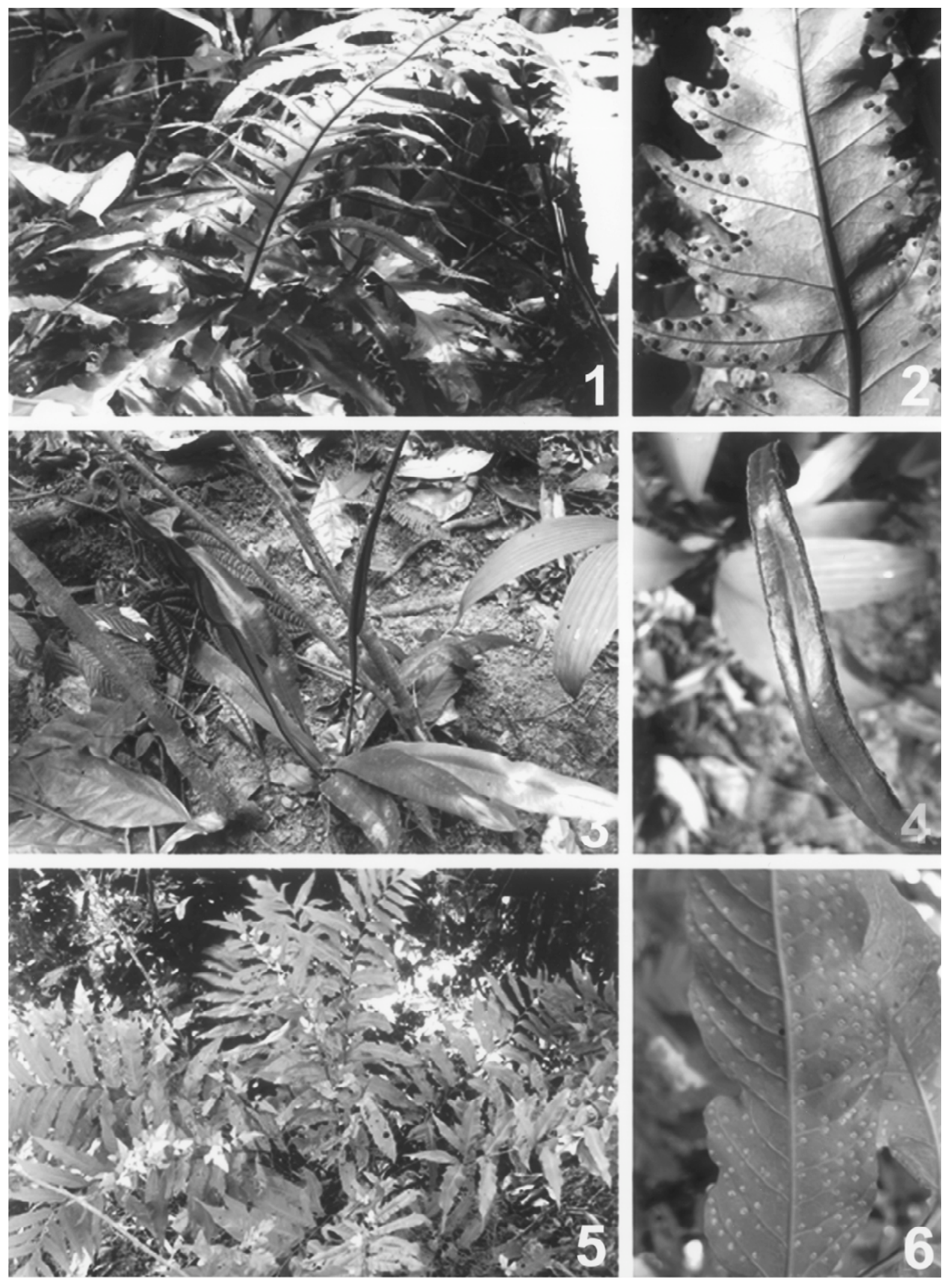

Figuras 1-2. Tectaria $\times$ michleriana. 1. En su hábitat. 2. Acercamiento de la lámina con soros.

Figuras 3-4. T. panamensis. 3. En su hábitat. 4. Acercamiento de la lámina con soros.

Figuras 5-6. T. incisa. 5. En su hábitat. 6. Acercamiento de la lámina con soros. Fotografías de Aniceto Mendoza R. 